\title{
Superhydrophobic to Superhydrophylic Biomimetic Poly(3-Hydroxybutyrate) Surfaces Made by Phase Inversion
}

\author{
Constancio González-Obeso 1,2,3,a. Wenlong Song ${ }^{1,2}$; \\ Miguel A. Rodriguez-Pérez ${ }^{3, b}$; João F. Mano ${ }^{1,2, c}$
}

\author{
13B's Research Group - Biomaterials, Biodegradables and Biomimetics, University of Minho, \\ Headquarters of the European Institute of Excellence on Tissue Engineering and Regenerative \\ Medicine, AvePark, 4806-909 Taipas, Guimarães, Portugal \\ ${ }^{2}$ ICVS/3B's - PT Government Associate Laboratory, Braga/Guimarães, Portugal \\ ${ }^{3}$ Cellular Materials Laboratory (CellMat), Condensed Matter Physics Department, University of \\ Valladolid, Facultad de Ciencias, Prado de la Magdalena s/n, 47011, Valladolid, Spain. \\ a constancito@gmail.com, b marrod@fmc.uva.es, ${ }^{\mathrm{c}}$ jmano@dep.uminho.pt
}

Keywords: Poly(3-hydroxybutyrate); superhydrophobic; superhydrophilic, phase inversion; biomaterial, plasma, contact angle modification

\begin{abstract}
Biodegradable and biocompatible materials have gained increased attentions because of their applications in biomedicine and tissue engineering. Among them, poly(3-hydroxybutyrate) (PHB), a natural origin polymer, has been employed in biomedical applications as a suitable substitute of synthetic polymers for preparing scaffolds and other devices. In this context it is relevant to understand the interactions between the cells and PHB surfaces, which are governed by aspects such as surface topography and chemical composition. Both, surface topography and chemistry determine surface wettability. The aim of this work is to process PHB surfaces exhibiting extreme wettablility properties, ranging from superhydrophobic (surface contact angle higher than $150^{\circ}$ ) to superhydrophilic (surface contact angle lower than $5^{\circ}$ ). A simple phase inversion method was used to fabricate surperhydrophobic PHB surface. Chloroform was used as solvent, and a mixture of water and ethanol as the coagulation bath. The water contact angle was found to be $154.3 \pm 2.9^{\circ}$. Furthermore the surface was treated by argon plasma for different times, permitting the control of wettability of the PHB surface from the superhydrophobic to superhydrophilic regime.
\end{abstract}

\section{Introduction}

Wetting is the ability of a drop of liquid to maintain contact with a surface. Surfaces having a contact angle (CA) lower than $150^{\circ}$, but higher than $90^{\circ}$, are called hydrophobic; and those having a CA higher than $150^{\circ}$ are called superhydrophobic. Due to the little interaction between the surface and the drop superhydrophobic surfaces have gained attention in many fields of science such as industrial, agriculture and biotechnology [1,2]. Interaction between the drop and the surface is mainly controlled by surface chemistry and topology. Wetting of a liquid onto an ideal flat surface is described by Young's equation:

$$
\gamma \cos \theta_{e}=\gamma_{S V}-\gamma_{S L}
$$

Being $\gamma$ the surface tension of the liquid, $\gamma_{S V}$ the surface tension between solid-air and $\gamma_{S L}$ the surface tension between solid-liquid [3]. This equation predicts the equilibrium CA between the liquid and the flat and smooth surface. Young's equation is only valid for perfect flat and smooth surfaces. Wenzel and Cassie-Baxter equations describe the CA of a drop of liquid onto a rough surface. The Wenzel model is used when the liquid is in contact with the surface in every point; Cassie-Baxter model is used in the case that air is trapped at some points between the surface and the liquid (Fig. 1). A rough surface is characterised by its roughness factor, $f$, that gives the ratio between the real surface and the projection of this surface onto a flat plane. 


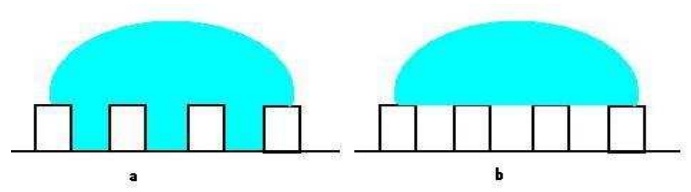

Figure 1. Wetting onto rough surfaces. a) Wenzel model. B) Cassie-Baxter model

Rough surfaces that wet according to the Wenzel model are described by the following condition for equilibrium:

$$
\cos \theta^{*}=\frac{a}{\gamma}=f \frac{\gamma_{S V}-\gamma_{S L}}{\gamma}=f \cos \theta_{e}
$$

Being $\theta^{*}$ the measured CA and $\theta_{\mathrm{e}}$ the CA that would be measured if the surface was perfectly flat. From this equation we see that roughness amplifies the tendency of the flat surface: if $\theta_{\mathrm{e}}>90^{\circ}$, then $\theta^{*}>\theta_{\mathrm{e}}$; if $\theta_{\mathrm{e}}<90^{\circ}$, then $\theta^{*}<\theta_{\mathrm{e}}[4]$.

For describing the Cassie-Baster wetting model ones consider that the surface is formed by two different materials, one would be the material itself and the other would be air bubbles trapped in cavities of the rough surface. In this case, the condition for equilibrium is:

$$
\gamma \cos \theta^{*}=\Phi\left(\gamma_{S V}-\gamma_{S L}\right)-(1-\Phi) \gamma
$$

Being $\Phi$ the fraction of surface occupied by the material and $\theta^{*}$ the CA measured. From this equation, we see that by modifying either the geometry of the surface or the surface energy we can modify $\theta^{*}$. Also from Eq.3 we see that sufficiently high roughness can lead to superhydrophobicity. We find in nature many examples of such superhydrophobic surfaces. One of these is the Lotus leaf [5]. Many studies have been made analysing the structure of the Lotus leaf, showing the importance of the double scale of roughness (micro and nano scale) for achieving superhydrophobicity $[6,7,8]$.

The spreading coefficient $(S)$ is used for describing the dynamical behaviour of the drop sitting on the surface. It is defined as:

$$
S=\gamma_{S V}-\gamma_{S L}-\gamma_{L V}
$$

If $S \leq 0$ the drop will stay still and will not spread; if $S>0$ the drop will spread. The bigger $S$ is, the faster the spreading is [9]. All equations presented above relate surface tension of the surface with the CA measured. Thus, modifying the surface tension will lead to changes in the CA and will affect the spreading coefficient $(S)$. Plasma treatment is a very useful tool to modify surface properties, such as its chemical composition [10], which could also have a positive effect in the biological response in biomaterials [11]. Moreover it has the advantages of being a dry, solvent-free and low consuming of chemical products [12].

Phase inversion was chosen as the method for preparing the PHB superhydrophobic surfaces. Phase inversion is a process where a polymer solution is forced to separate in two phases: one with a high polymer concentration (making the polymer to precipitate) and other with a low polymer concentration [13]. Phase inversion can be used in the preparation of superhydrophobic surfaces from different polymers $[14,15,16]$. In this case, a liquid-liquid demixing process by immersion was used to induce phase separation. The solution, containing the polymer and the solvent (S) is immersed into a coagulation bath, where the non-solvent (NS) exchanges with the solvent, making the polymer to precipitate. Different structures can be achieved, depending on how fast the liquidliquid demixing process is, i.e, how fast phase inversion is induced. The faster the process is, smaller spherical particles are formed. As the process slows down, bicontinuous morphologies appear, due to spinodal decomposition [17]. 


\section{Materials and Methods}

Polyhydroxybutyrate (PHB) was supplied by Biomer. This PHB is obtained from bacteria and was in the form of a very thin white powder. Chloroform was supplied by Carlo Erba Reagents, with a purity of $99 \%$. Ethanol was supplied by PANREAC, with a purity of $99.5 \%$.

A $15 \% \mathrm{w} / \mathrm{v}$ PHB in chloroform solution was steered for at least 12 hours and a mixture of 50-50\% in volume of ethanol and water was used as the coagulation bath. About $200 \mu 1$ of solution was carefully poured in to the coagulation bath with a Pasteur pipette. In this case the end of the pipette is immersed in the coagulation bath and placed $1 \mathrm{~cm}$ above the bottom of the recipient. Phase inversion took place as the solution was poured in to the coagulation bath. During this process phase inversion takes place first in the surface of the drop of solution, making PHB to precipitate rapidly forming an "upper film". When phase inversion has totally occurred (after at least 12 hours) samples are taken out from the coagulation bath. The "upper film" can be easily removed with water. The surface under the "upper film" is superhydrophobic after being dried out in air (Fig. 2).
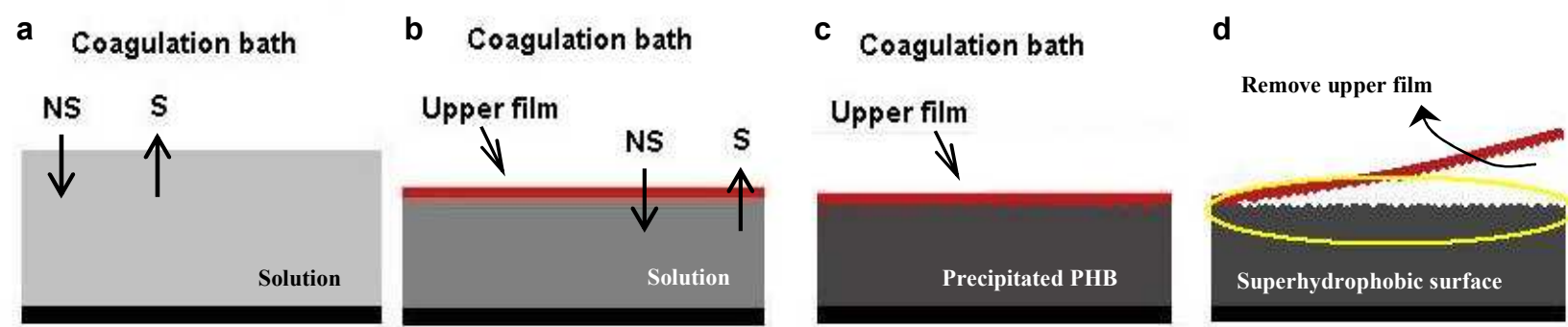

Figure 2. Scheme of the preparation of PHB superhydrophobic surfaces using a phase inversion methodology. a) the presence of the non solvent induces the formation of a solution of the two liquids, making the solution to supersaturate and PHB to precipitate. b) Formation of the "upper film" while phase inversion continues to take place. c) PHB sample before taking out of the coagulation bath. d) After removing the "upper film" and air drying, the surface shows superhydrophobic characteristics.

The composition of the coagulation bath is crucial for obtaining the superhydrophobic surface. Due to the great miscibility of water and ethanol, the coagulation bath is a homogeneous mixture. Water and ethanol possess lower densities than chloroform, so as we pour the solution into the coagulation bath it settles down in the bottom. Due to the low miscibility of chloroform and water (less than $0.8 \mathrm{~g} / 100 \mathrm{ml}$ ), as de drop settles down in the bottom it doesn't spread, forming rounded flat drops of solution. Furthermore, as ethanol is very soluble in water and chloroform, ethanol from the coagulation bath can be quickly exchanged with the chloroform of the solution, making the phase inversion to take place, and PHB to precipitate forming spherical particles.

CA was measured with a contact angle meter (from Dataphysics, model OCA15+), using a $5 \mu 1$ drop of HPLC water. At least 3 samples were used in each measurement. All measurements were done at room temperature. Argon plasma treatment was applied for different times $(0,20,40,60,80,90$, 100, 120, 160 and 180 seconds) using a plasma treatment equipment from Gala Instrument, model Plasma Prep5. The pressure was kept always between 0.16 and $0.2 \mathrm{mbar}$, and the voltage applied was $2.2 \mathrm{~V}$. 


\section{Results and Discussion}

PHB is a hydrophobic polymer, since the CA of a flat smooth surface (Fig.3a) obtained by casting a $15 \% \mathrm{w} / \mathrm{v}$ PHB in chloroform solution is $107.4 \pm 2.2^{\circ}$ (Fig 3b). As described before, during the selected procedure to prepare superhydrophobic PHB surfaces, a fast phase inversion process leads to the formation of nano and micrometric spherical particles that settle down, forming a rough surface with two length of roughness (Fig.3c). This surface configuration leads to superhydrophobic substrate with a CA of $154.3 \pm 2.9$ (Fig.3d).

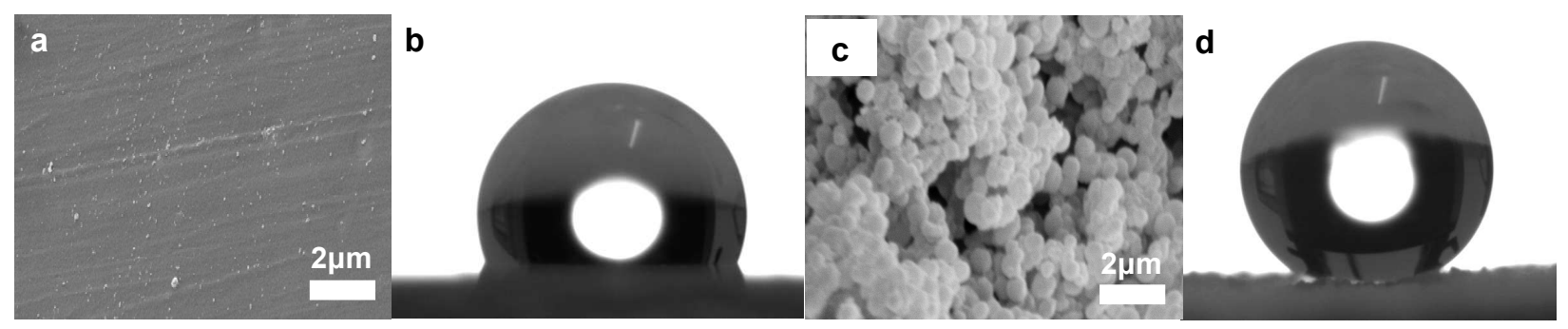

Figure 3.(a)SEM image of PHB flat surface, (b) picture of a $5 \mu 1$ drop of water sitting on PHB flat surface, (c) SEM image of superhydrophobic PHB surface, (d) picture of a $5 \mu 1$ drop of water sitting on superhydrophobic PHB.

The use of energetic radiations such as plasma or UV/ozone, may modify the wettability of superhydrophobic surfaces down to the superhydrophilic regime [18, 19]. Such kind of surface treatment was used in the developed PHB substrates. After argon plasma treatment, the CA of the PHB rough substrates was measured as a function of the exposure time (Fig. 4). We can observe that $\mathrm{CA}$ decreases as plasma treatment time increases. We observe two different behaviours. First, from 0 to 80 seconds, a progressive slow decrease in CA as plasma treatment time increases. For longer treatments, the CA rapidly decreases to values near to 0 (superhydrophilic behaviour).

This abrupt change in the behaviour of CA points to a change in the $S$ coefficient, that should influence the contact angle of the water drop measured at the employed time scale. This is, for treatments under 100 seconds $S$ seems to be negative (this means no spreading of the drop) and positive for treatments above 100 seconds. It is also notable that, although CA gets to really low values for plasma treatments longer than 100 seconds, the behaviour of the drop is not the same in all cases. The longer the plasma treatment is the drop spreads faster. Fig. 5 quantifies this effect by following the change of the CA of droplets deposited in surfaces with different treatment times. This behaviour suggests that, as plasma treatment increases above 100 seconds, the spreading coefficient gets higher positive values.

From Fig. 5 we see that, for the PHB superhydrophobic surface treated for 120seconds, CA reaches values under $10^{\circ}$ in 520 seconds. For 160 seconds of plasma treatment, it takes 400 seconds and for 180seconds of treatment it just takes 90 seconds. From this data it is clear that plasma treatment affects the spreading of the drop. 


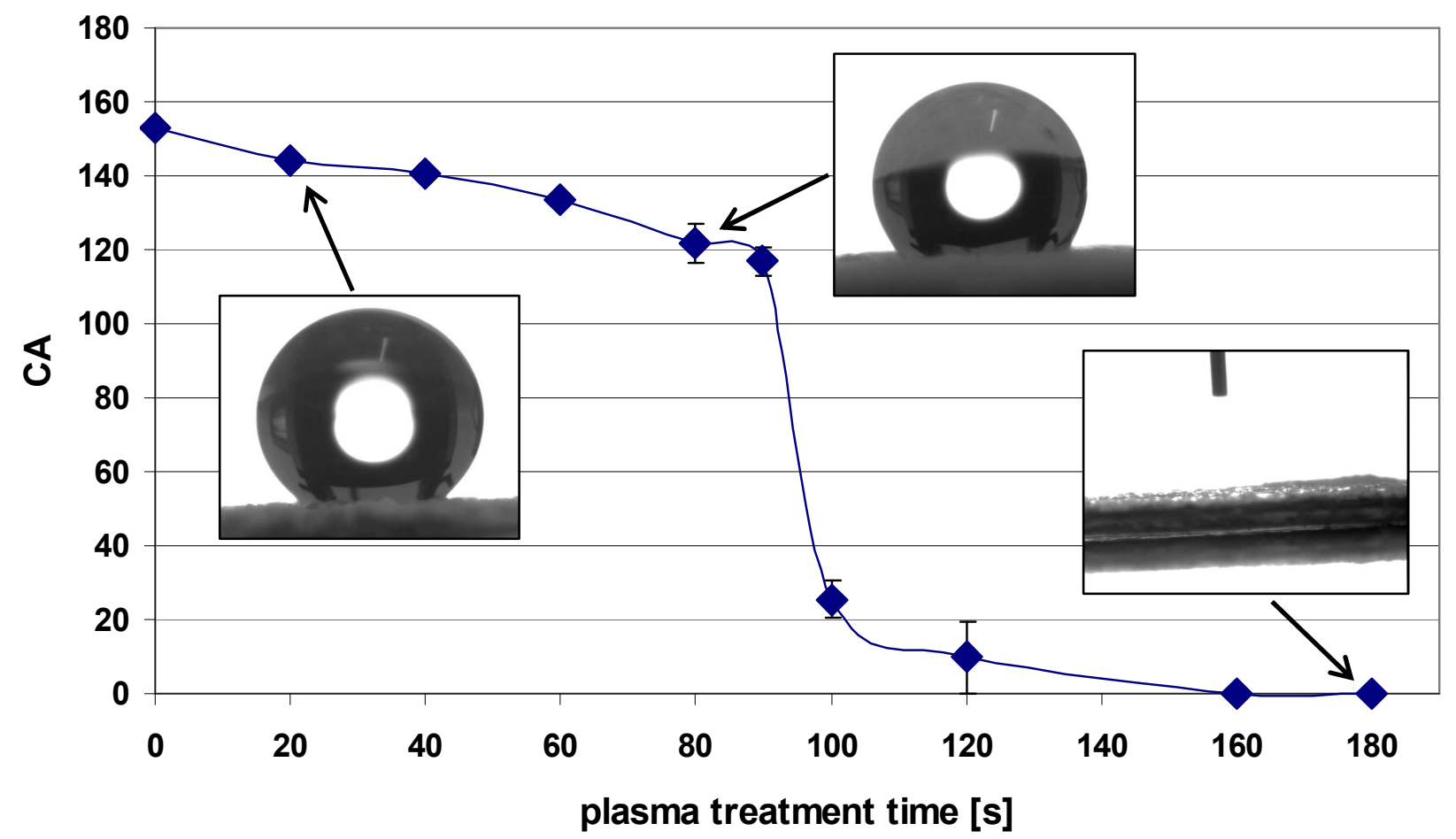

Figure 4. Graphical representation of the variation of CA with plasma treatment time

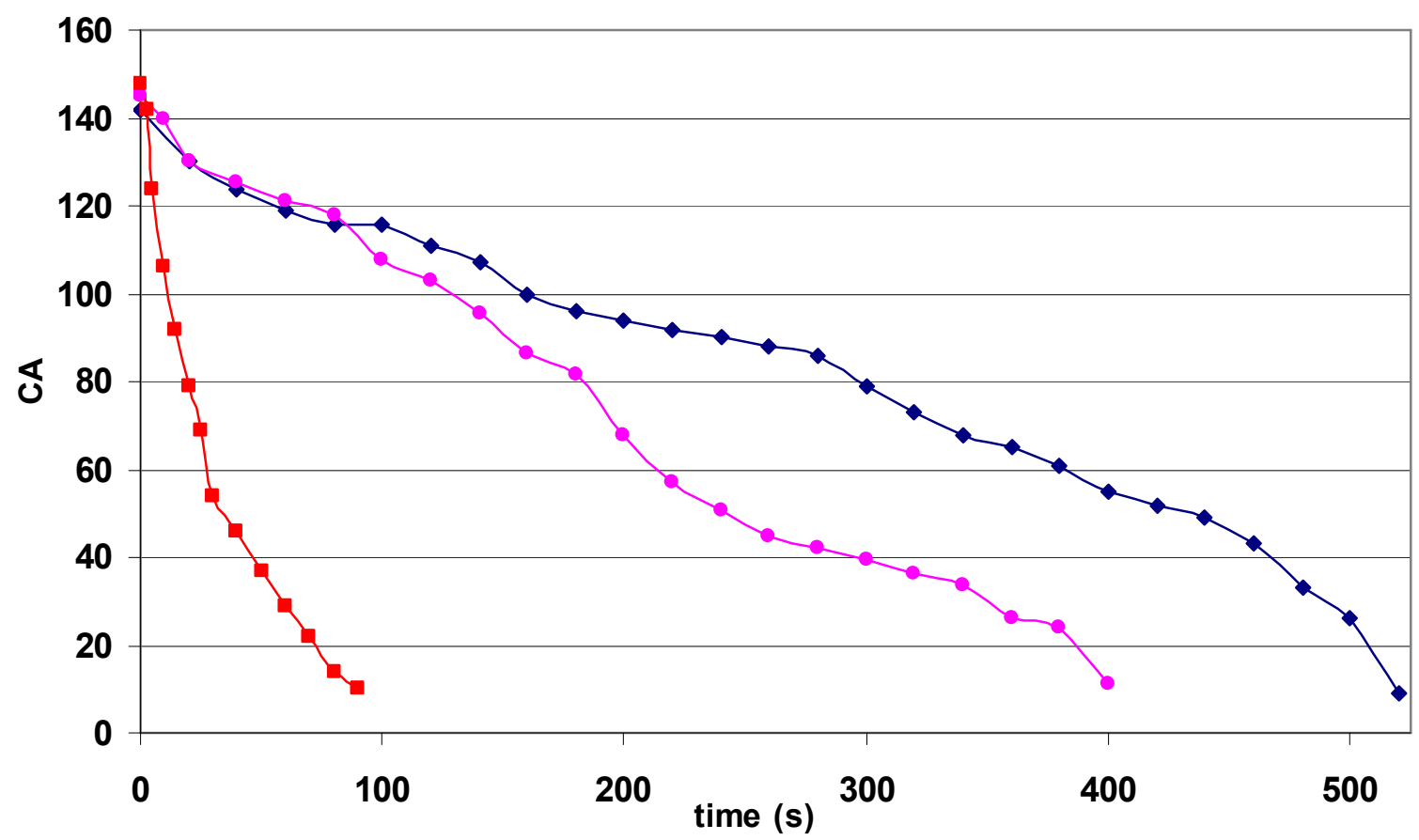

Figure 5. Graphical representation of the variation of the CA with time of an $8 \mu 1$ drop of water sitting into different time treated surfaces: $\bullet 120$ seconds of treatment, $\bullet 160$ seconds of treatment, 180 seconds of treatment.

\section{Conclusions}

From this work we can conclude that PHB superhydrophobic surfaces can be fabricated by a simple phase inversion method. CA can be controlled from superhydrophobic to superhydrophilic $\left(\mathrm{CA}<5^{\circ}\right)$ using plasma treatment. Also the dynamical behaviour of the drop can be controlled with plasma treatment, obtaining a faster spreading of the drop for longer plasma treatment. 


\section{References}

[1] Z. Yuan, H. Chen, J. Tang, X. Chen, D. Zhao, Z. Wang, Facile method to fabricate stable superhydrophobic polystyrene surface by adding ethanol, Surf. \& Coat. Tech. 201 (2007) 71387142.

[2] P. Roach, N.J. Shirtcliffe, M.I. Newton, Progess in superhydrophobic surface development, Soft. Matter 4 (2008) 224-240.

[3] G. Whyman, E. Bormashenko, T. Stein, The rigorous derivation of Young, Cassie-Baxter and Wenzel equations and the analysis of the contact angle hysteresis phenomenon, Chem. Phys. Let. 450 (2008) 355-359.

[4] D. Quéré, Non-sticking drops, Rep. Prog. Phys. 68 (2005) 2495-2532.

[5] Z. Guo, W. Liu, B.L. Su, Superhydrophobic surfaces: From natural to biomimetic to functional, J. Coll. Interf. Sci. 353 (2011) 335-355.

[6] L. Gao, T.J. McCarthy, The "Lotus effect" explained: tow reasons why tow length scales of topography are important, Langmuir 22 (2006) 2966-2967.

[7] B. Bhushan, Y. C. Jung, Wetting study of patterned surfaces for superhydrophobicity, Ultramicroscopy 107 (2007) 1033-1041

[8] S.H. Sajadinia, F.Sharif, Thermodynamic analysis of the wetting behaviour of dual scale patterned hydrophobic surfaces, J. Coll. Interf. Sci. 344 (2010) 575-5838.

[9] W.A Zisman, Relation of the equilibrium contact angle to liquid and solid constitution, in Contact Angle, Wettability and Adhesion, F. Fowkes, Advances in Chemistry, American Chem. Soc. 1964 pp. 1-51.

[10] P.K. Chu, J.Y. Chen, L.P. Wang, N. Huang, Plasma-surface modification of biomaterials, Mat. Sci. Eng. 36 (2002) 143-206.

[11] S.S. Silva, S.M. Luna, M. E. Gomes, J. Benesch, I. Pashkuleva, J.F. Mano, R.L. Reis, Plasma Surface Modification of Chitosan Membranes: Characterization and Preliminary Cell Response Studies, Macromol. Biosci. 8 (2008) 568-576.

[12] S. Guruvenket, G.M. Rao, M. Komath, A.M. Raichur, Plasma surface modification of polystyrene and polyethylene, App. Surf. Sci. 236 (2004) 278-284.

[13] E. Reverchon, R. Adami, G. Caputo, I. De Marco, Spherical microparticles production by supercritical antisolvent precipitation: Interpretation of results, J. of Supercrit. Fluids 47 (2008) 7084.

[14] N.M. Alves, J. Shi, E. Oramas, J.L. Santos, H. Tomás, J.F. Mano, Bioinspired superhydrophobic poly(L-lactic acid) surfaces control bone marrow derived cells adhesion and proliferation, J. Biomed. Matter. Res. A. 91 (2009) 480-488.

[15] W. Song, V. S. Gaware, Ö. V. Rúnarsson, M. Másson, J.F. Mano, Functionalized superhydrophobic biomimetic chitosan-based films, Carbohy. Polym. 81 (2010) 140-144.

[16] N.M. Oliveira, A.I. Neto, W. Song, J.F. Mano, Two-dimensional open microfluidic devices by tuning the wettability on patterned superhydrophobic polymeric surface, Appl. Phy. Express 3 (2010) 085205.

[17] P. van de Witte, P.J. Dijkstra, J.W.A. van den Berg, J. Feijen, Phase separation processes in polymer solutions in relation to membrane formation, J. Membr. Sci. 117 (1996) 1-31.

[18] W. Song, D.D. Veiga, C.A. Custódio, J.F. Mano, Superhydrophobic coatings: bioinspired degradable substrates with extreme wettability properties, Advan. Matter. 21 (2009) 1830-1834.

[19] A.I. Neto, C.A. Custódio, W. Song, J.F. Mano, High-throughput evaluation of interactions between biomaterials, proteins and cells using patterned superhydrophobic substrates, Soft. Matter 7 (2011) 4147-4151. 